\title{
Open versus robotic-assisted partial nephrectomy: a multicenter comparison study of perioperative results and complications
}

\author{
Gianni Vittori
}

Received: 1 April 2013/Accepted: 20 July 2013/Published online: 4 August 2013

(C) Springer-Verlag Berlin Heidelberg 2013

\begin{abstract}
Purpose To compare surgical results, morbidity and positive surgical margins rate of patients undergoing robotic partial nephrectomy (RPN) versus open partial nephrectomy (OPN).

Methods This is an observational multicenter study promoted by the "Associazione GIovani Laparoscopisti Endoscopisti” (AGILE) no-Profit Foundation, which involved six Italian urologic centers. All clinical, surgical, and pathological variables of patients treated with OPN or RPN for renal tumors were gathered in a prospectively maintained database. Tumor nephrometry was measured with PADUA score, and complications were stratified with modified Clavien system. Differences between RPN and OPN group were assessed with univariate analysis. Perioperative variables independently associated with complications were assessed with multivariate analysis.

Results A total of 198 and 105 patients were enrolled in OPN and RPN group, respectively. Both had similar demographics, indications to surgery, tumor nephrometry, renal function, WIT (18.7 vs. $18.2 \mathrm{~min} ; p=\mathrm{NS}$ ), positive margin rate (5.6 vs. $5.7 \% ; p=\mathrm{NS}$ ), intraoperative complications, and postoperative medical complications. Compared to OPN, RPN group was significantly more morbid $(p=0.04)$, included tumors with smaller size

$(p=0.002)$, had longer operative time $(p<0.001)$, lower

G. Vittori $(\square)$

Clinica Urologica I, Azienda Ospedaliera Careggi,

Università di Firenze, Villa Monna Tessa,

Viale Pieraccini 18, 50139 Florence, Italy

e-mail: giannivittori@yahoo.it

blood loss, surgical postoperative complications (5.7 vs. $21.2 \%, p<0.001)$, Clavien 3-4 surgical complications (1 vs. $9.1 \%, p=0.001)$, and shorter hospitalization. The surgical approach resulted independently correlated with surgical complications on multivariate analysis.

Conclusion In the present series, RPN was associated with a significant reduction of blood loss, surgical complications, including the reintervention rate for urinary fistula and postoperative bleeding, and with a shorter hospitalization.

Keywords Kidney tumor - Partial nephrectomy · Robotics · Complications

$\begin{array}{ll}\text { Abbreviations } & \\ \text { I postop./III postop. } & \text { First/third postoperative day } \\ \text { ASA } & \begin{array}{l}\text { American Society of } \\ \text { Anaesthesiologists }\end{array} \\ \text { BMI } & \text { Body mass index } \\ \text { EBL } & \text { Estimated blood loss (during } \\ & \text { surgery) } \\ \text { ECOG } & \text { Eastern cooperative oncology group } \\ \text { IQR } & \text { Interquartile range } \\ \text { LOS } & \text { Length of hospital stay } \\ \text { OPN } & \text { Open partial nephrectomy } \\ \text { PB } & \text { Postoperative bleeding } \\ \text { PN } & \text { Partial nephrectomy } \\ \text { PSM } & \text { Positive surgical margins } \\ \text { RPN } & \text { Robotic partial nephrectomy } \\ \text { RCC } & \text { Renal cell carcinoma } \\ \text { RR } & \text { Related risk } \\ \text { SD } & \text { Standard deviation } \\ \text { UCS } & \text { Urinary collecting system } \\ \text { UF } & \text { Urinary fistula } \\ \text { WIT } & \text { Warm ischemia time }\end{array}$
\end{abstract}




\section{Introduction}

Open partial nephrectomy (OPN) is the standard treatment for renal masses up to $7 \mathrm{~cm}$ in major dimensions, if technically feasible [1,2], since long-term oncological findings resulted comparable to those of radical nephrectomy [3], and the preservation of renal function associated with partial nephrectomy (PN) resulted of crucial importance to prevent all-cause mortality [4, 5]. Laparoscopic partial nephrectomy (LPN) and robotic partial nephrectomy (RPN) are the main mini-invasive alternatives to OPN, aimed at reducing the added morbidity of an open incision and potential rib resection. While bringing excellent results in experienced hands, the diffusion of LPN has been limited by the technical difficulty and is currently performed in a few high-volume centers. At contrary, RPN seemed to be a more reproducible technique, able to bridge the technical difficulties of LPN in favor of a broader diffusion of minimally invasive PN. A few well-designed comparative studies have investigated the perioperative outcomes of RPN versus those of LPN [6-8], while to date no prospective studies comparing RPN and OPN are available, but only few retrospective series with limited cases [9-11]. The aim of the present study is to compare the perioperative results and surgical complications of RPN and OPN.

\section{Patients and methods}

This is a two-year observational multicenter study promoted by the "Associazione GIovani Laparoscopisti Endoscopisti" (AGILE) no-profit foundation, which includes a group of urologists performing in daily practice traditional, laparoscopic, and robotic surgery in different hospitals. Between January 2010 and December 2011, the clinical records of all patients consecutively treated with OPN or RPN for clinically localized renal cell carcinoma (RCC) were gathered at six Italian urological centers (Florence, Udine, Brescia, Abano Terme-PD, Novara, Mantova) and included in a prospectively maintained database. The approach selection was based on a chronological criterion, as the robot was available on 4 centers at the beginning of the study and in each center at the end. Additional criteria were patients with preferences for specific approach, or with significant previous transperitoneal surgery, which have been treated with OPN. Approval of study protocol by the local ethical committee was obtained at each center, and informed consent was collected for all patients.

Surgical technique and surgeons experience

The surgical resection was done by standard partial nephrectomy, leaving a minimal tumor-free surgical margin around RCC. Tachosil ${ }^{\circledR}$ or Floseal ${ }^{\circledR}$ plus surgical bolsters $\left(\right.$ Tabotamp ${ }^{\circledR}$ ) were the hemostatics used in all centers. The surgical approach was chosen according to surgeons and centers preferences as well as to the da Vinci system availability. The surgical complexity and nephrometry score did not represent criteria for the decision. At the beginning of the study, all surgeons performing RPN were within the learning curve (20 cases) [12], but they had previous experience in LPN, while the surgeons performing OPN had a substantial previous experience in open kidney surgery ( $>50$ cases).

Robotic partial nephrectomy was done with da Vinci S/Si Surgical Systems, (Intuitive Surgical, Sunnyvale, USA), all but one center (Abano Terme-Padua) used a transperitoneal approach, because it was the preferred and commonly used access. Patients were placed in flank position. A standard 3-arm approach with a fourth trocar for the assistant and fifth trocar as needed for right-sided cases was used. The renal hilum was identified to locate renal artery and vein. In case of unfavorable position, the kidney was isolated and rotated in order to perform comfortably the demolitive and reconstructive phases. Ischemia, if needed, was obtained with the clamping of renal artery or, according to surgeon's preference, with a selective arterial clamping. Hemostasis in the resection bed was achieved with running sutures (Monocryl 3-0) preloaded with a Hem-o-lok, brought outside through the parenchyma according to the sliding-clip technique. Care was taken to repair all visible opened calices and bleeding sites with these running sutures. Usually, the cortical defect was closed with horizontal interrupted sutures after apposition of hemostatic agents.

Open partial nephrectomy was done using a lateral retroperitoneal approach for all patients. The renal pedicle was usually controlled en bloc with a vascular clamp. Alternatively, manual compression was used in case of polar lesions according to center's preference. Since the warm ischemia time (WIT) was usually under $25 \mathrm{~min}$, cold ischemia was never used in this series. Bleeding vessels and incidentally opened calyces were sutured with 4-0 monofilament running sutures. The parenchymal defect was closed with horizontal interrupted sutures after apposition of hemostatic agents.

\section{Data collection}

The following patients characteristics were included in the database: age, gender, body mass index (BMI), American Society of Anesthesiologists (ASA) score, Charlson index, and surgical indication. All patients were staged preoperatively with computed tomography or magnetic resonance imaging. The nephrometry of tumors was assessed by the clinical imaging and measured with PADUA score [13]. The use of pedicle clamping, warm ischemia time (WIT), total operative time, estimated blood loss (EBL), length of hospital stay (LOS), including the date of surgery, was 
registered. The blood hemoglobin and serum creatinine were recorded preoperatively, in first and third postoperative day. The estimated glomerular filtration rate (eGFR) was calculated with the modification of diet in renal disease equation [14]. Pathological tumor size, 2009 TNM stage [15], Fuhrman nuclear grade [16], surgical margin status, and histological subtypes according to World Health Organization classification [17] were registered. No central pathological slide review was performed. All the intra- and postoperative medical and surgical complications, occurring during surgery and within 30 days, were recorded. The severity of surgical complications was stratified according to the modified Clavien system [18]. Postoperative blood loss was registered in case of reduction $>2.5 \mathrm{~g} / \mathrm{dL}$ in hemoglobin level (grade 1), and the need of blood transfusions (grade 2), superselective embolization of renal artery (grade 3a) or of re-intervention (grade 3b-4) was listed. Urinary fistula was registered if there was persistent leakage from the drainage beyond sixth postoperative day and a biochemical analysis of the drain fluid consistent with urine (drainage fluid-to-serum creatinine ratio greater than 2). Ureteral stenting was limited to urinary fistula with persistent leakage beyond tenth postoperative day.

\section{Statistical analysis}

Continuous parametric variables are presented as mean \pm standard deviation (SD), nonparametric variables as median and interquartile range (IQR), and categorical variables with frequencies and proportions. PADUA score was analyzed both as nonparametric continuous (3-13) variable and as a binary with cutoff $10(<10$ vs. $\geq 10)$. Univariate analysis (Pearson's $\chi^{2}$ test, unpaired $t$ test, and Mann-Whitney $U$ test, as appropriate) was used to assess the differences of preoperative variables, surgical results, and complications between RPN and OPN group. A multivariate logistic regression model tested the ability of the surgical approach, along with the other relevant clinical variables, to predict overall surgical complications and Clavien grade 3-4 surgical complications, analyzed separately. All tests were two-sided, with a statistical significance at $p<0.05$. Analyses used all events in Stat view 5.0.1 (SAS Institute Inc. Cary, MC, USA).

\section{Results}

Perioperative data are shown in Table 1.

Baseline characteristics

Overall, 302 patients were included. Of them, 198 underwent OPN and 105 RPN. The OPN and RPN groups were significantly different in the following preoperative parameters: clinical tumor size $(3.5 \pm 1.8$ and $2.8 \pm 1.5$; $p=0.002)$ and Charlson index $\geq 2$ (5.6 and $25.7 \%$; $p=0.04)$, while imperative indications had a trend of difference that did not reach the statistical significance $(5$ versus $1 \%, p=0.07$ ).

Intra- and postoperative outcomes

Clamping of renal vessels was used in $48 \%(95 / 198)$ of OPN patients and in $62 \%(65 / 105)$ of RPN patients. When used, WIT resulted not significantly different in OPN versus RPN group (18.7 and $18.2 \mathrm{~min}$, respectively). In OPN group, the operative time was significantly lower $(p<0.0001)$ and EBL was higher $(p=0.0004)$. One robotic procedure was converted to open due to hemorrhage, while the total number of intraoperative complications did not differ between groups. In third postoperative day, the mean change of hemoglobin was higher in OPN group ( $p=0.0019$ ), while no significant difference resulted in the mean variation of eGFR. Overall, postoperative complications occurred in 48/198 (24.2\%) patients in OPN group and in 9/105 $(8.6 \%)$ in RPN group $(p=0.009)$. About $3 \%$ of patients had medical complications in each group, thus leaving a higher rate of surgical complications in OPN versus RPN group ( 21.2 vs. $5.7 \%$; $p<0.0001)$. The stratification for severity of surgical complications is summarized in Table 2. Postoperative bleeding (PB) and urinary fistula (UF) were the most frequent; in OPN group, PB occurred in 29/198 (14.6\%) patients; in 21/198 $(10.6 \%)$, it was managed with transfusions and in $7 / 198$ $(3.5 \%)$ with a second procedure under anesthesia; of these, 4/198 (2\%) were subjected to a superselective embolization and $3 / 198(1.5 \%)$ to a reintervention. Nephrectomy was necessary for two patients (1\%), due to the impossibility to stop the bleeding from the resection bed. In OPN group, the UF occurred in $11 / 198$ (5.5\%) patients. Of these, 2/198 (1\%) were treated with expectancy, 8/198 (4\%) with the ureteral stent, and one case $(0.5 \%)$ was complicated with renal abscess and required nephrectomy. Considering the surgical complications of the RPN group, PB occurred in 5/105 (4.8\%) of patients; in 4/105 (3.8\%), it was managed with transfusions only, and one patient (1\%) underwent a superselective embolization. In RPN group, UF occurred in 1/105 (1\%) patients and was treated conservatively; no ureteral stenting was needed in robotic group. No Clavien grade 5 complication occurred in either group.

Then, we assessed the preoperative variables associated with surgical complications (Table 3). The clinical tumor size, the Charlson index, the surgical indication, the PADUA score, and the surgical approach were significantly associated with postoperative surgical complications 
Table 1 Perioperative data of patients and tumors, stratified according to surgical procedure

Open partial nephrectomy

$N=198$

Preoperative variables

Male gender $(\%)$

Age (years) mean $\pm \mathrm{SD}$

Body mass index median (IQR)

ASA score $3(\%)$

Charlson index $\geq 2(\%)$

Imperative indication (\%)

Clinical tumor size mean $\pm \mathrm{SD}$

PADUA score median (IQR)

No pts. with PADUA $\geq 10(\%)$

Preoperative $\mathrm{Hb}(\mathrm{g} / \mathrm{dL})$ mean $\pm \mathrm{SD}$

Preoperative serum creatinine $(\mathrm{mg} / \mathrm{dL})$ mean $\pm \mathrm{SD}$

Preoperative eGFR ( $\mathrm{ml} / \mathrm{min})$ mean $\pm \mathrm{SD}$

Intraoperative variables

Warm ischemia time (min) mean $\pm \mathrm{SD}$

Operative time (min) mean $\pm \mathrm{SD}$

Estimated blood loss (cc) mean \pm SD

Intraoperative complications (\%)

Conversion to open surgery

Clamping of the renal pedicle

Pedicle en bloc

Artery

Selective clamping of artery branch

No clamping

Surgical results and complications

Variation $\mathrm{Hb}$ (preoperative-III postoperative) (g/dL)

Variation (III postoperative-preoperative) serum creatinine

Variation eGFR (preoperative-III postoperative) $\mathrm{ml} / \mathrm{min}$

Overall postoperative complications (pts)

Surgical postoperative complications (pts)

Postoperative bleeding

Urinary fistula

Intestinal lesions

Medical postoperative complications (pts)

Cardiovascular

Respiratory

Clavien grade 3-4 surgical complications (pts)

Length of hospital stay, including the date of surgery

Days median (IQR)

Pathological data

Pathological histotype (\%)

Benign

Malignant

Clear cell

Papillary

Chromophobe

Other malignant
62

$63.8 \pm 12.4$

$26.2(24.3-29.3)$

14.6

5.6

5.05

$3.5 \pm 1.8$

7 (7-8)

7.1

$13.9 \pm 1.4$

$1.0 \pm 0.4$

$79.0 \pm 21.1$

$18.7 \pm 8.1$

$123 \pm 43$

$230 \pm 208$

5.1

$95(48 \%)$

$95(48 \%)$

$103(52 \%)$

$3.1 \pm 1.9$

$0.2 \pm 0.3$

8.3

$48 / 198(24.2) \%$

$42 / 198$ (21.2) \%

$29(14.6 \%)$

$11(5.6 \%)$

$2(1 \%)$

6/198 (3.0) \%

$4(2 \%)$

2 (1\%)

18/198 (9.1) \%

7 (5-10)
Robotic partial

$p$

nephrectomy

$N=105$

$65.8 \quad$ Ns

$62.3 \pm 11.6 \quad \mathrm{Ns}$

$25.7(24.0-27.7) \quad$ Ns

$14 \quad$ Ns

$\begin{array}{ll}25.7 & 0.04\end{array}$

$1 \quad 0.07$

$2.8 \pm 1.5 \mathrm{~cm} \quad 0.002$

$7(6-7) \quad$ Ns

$6.7 \quad$ Ns

$14.1 \pm 1.2 \quad$ Ns

$0.9 \pm 0.2 \quad$ Ns

$89 \pm 20.8 \quad$ Ns

$18.2 \pm 7 \quad$ Ns

$168 \pm 56 \quad<0.0001$

$125 \pm 128 \quad 0.0004$

$2.9 \quad \mathrm{Ns}$

$1 / 1051 \%$

$65(62 \%) \quad$ Ns

$53(50.5 \%)$

$12(11.5 \%)$

$40(38 \%)$

$2.3 \pm 1.5$

0.0019

$0.3 \pm 0.1$

Ns

7

$9 / 105(8.6) \%$

Ns

0.009

$6 / 105(5.7) \%$

$<0.0001$

$5(4.8 \%)$

$1(1 \%)$

0

3/105 (2.9) \%

Ns

$3(2.9 \%)$

1/105 (1) \%

0.001

5 (4-6)

0.042

$14(13.3 \%)$

NS 
Table 1 continued

Open partial nephrectomy

$N=198$
Robotic partial nephrectomy $N=105$

\section{pT 2009 \\ pT1a \\ pT1b \\ pT2a-b \\ pT3a}

Fuhrman nuclear grade (\%)

G1

G2

G3-G4

Positive surgical margin

\author{
$112(56.6 \%)$ \\ $24(12.2 \%)$ \\ $8(4.0 \%)$ \\ $12(6.0 \%)$
}

$28(14.2 \%)$

$105(53.0 \%)$

$23(11.6 \%)$

$11(5.6 \%)$

\author{
$73(69.5 \%)$ \\ $7(6.6 \%)$ \\ $1(1 \%)$ \\ $10(9.6 \%)$ \\ $23(21.9 \%)$ \\ $55(52.4 \%)$ \\ $13(12.4 \%)$ \\ $6(5.7 \%)$
}

Univariate analysis with Pearson's chi-square test, unpaired $t$ test, and Mann-Whitney $U$ test, as appropriate

Table 2 Stratification for severity of postoperative surgical complications of RAPN versus OPN group with modified Clavien system

${ }^{\text {a }}$ One case of inadvertent lesion of the descendent colon in a kidney extensively attached to the peritoneum. One early strangulated bowel herniation through the lombotomic incision

\begin{tabular}{lll}
\hline Postoperative surgical complications & $\begin{array}{l}\text { Open partial } \\
\text { nephrectomy }\end{array}$ & $\begin{array}{l}\text { Robotic partial } \\
\text { nephrectomy }\end{array}$ \\
\hline Clavien-Dindo grade 1 & $3(1.5 \%)$ & $1(1 \%)$ \\
Drainage leakage treated with expectancy & $2(1 \%)$ & $1(1 \%)$ \\
Slight blood loss treated with bedrest & $1(0.5 \%)$ & 0 \\
Clavien-Dindo grade 2 & $21(10.6 \%)$ & $4(3.8 \%)$ \\
Blood loss treated with homologous blood transfusions & $21(10.6 \%)$ & $4(3.8 \%)$ \\
Clavien-Dindo grade 3 & $15(7.6 \%)$ & $1(1 \%)$ \\
Urinary fistula treated with ureteral stenting & $8(4 \%)$ & 0 \\
Blood loss treated with superselective hembolization & $4(2 \%)$ & $1(1 \%)$ \\
Reintervention due to hemorrhage & $1(0.5 \%)$ & 0 \\
Intestinal lesions treated with reintervention & $2(1 \%)^{\mathrm{a}}$ & 0 \\
Clavien-Dindo grade 4 & $3(1.5 \%)$ & 0 \\
Nephrectomy due to hemorrhage & $2(1 \%)$ & 0 \\
Nephrectomy due to renal abscess & $1(0.5 \%)$ & 0 \\
& 2 & \\
\hline
\end{tabular}

at the univariate analysis (data not shown), while the avoidance of pedicle clamping was not correlated with postoperative surgical complications $(p=0.59)$. On the multivariate analysis, only the imperative indication $(\mathrm{RR}=3.9 ; p=0.050)$ and the open surgical approach $(\mathrm{RR}=3.8 ; p=0.005)$ resulted independently associated with postoperative surgical complications. Finally, surgical approach $(\mathrm{RR}=9.4 ; p=0.03$ ) was the only variable independently associated with Clavien grade 3-4 surgical complications.

\section{Discussion}

In recent years, many papers compared the perioperative results of RPN and LPN, and current evidence suggests that RPN can reproduce the advantages of mini-invasiveness with a shorter learning curve, since it entails excellent perioperative outcomes after about 30 cases [12]. In some studies, it was found that RPN may decrease WIT, operative time and EBL [7, 19, 20], and in a recent series also the incidence of complications, compared to LPN [6].

Ideally, every surgical option for the conservative treatment of renal tumors should be compared to OPN that is the matching standard of treatment and has the robustness of data regarding surgical and oncological results [21]. To our knowledge, this is the second prospectively derived comparative study of perioperative outcomes of RPN and OPN. In accordance with our results, in the previous study, Simhan et al. [9] reported after RPN comparable WIT and eGFR modification, with shorter hospitalization. These data suggest that RPN may replicate the rapidity of the open approach in the more complex phase of intervention, also leading to comparable early functional results. 
Table 3 Multivariate analysis (logistic regression) of preoperative variables to predict surgical complications and Clavien grade 3-4 surgical complications after partial nephrectomy

\begin{tabular}{|c|c|c|c|c|c|c|}
\hline \multirow[t]{2}{*}{ Preoperative variable } & \multicolumn{3}{|c|}{ Postoperative surgical complications } & \multicolumn{3}{|c|}{ Postoperative Clavien-Dindo grade 3-4 surgical complications } \\
\hline & Risk ratio & $p$ value & $95 \% \mathrm{CI}$ & Risk ratio & $p$ value & $95 \% \mathrm{CI}$ \\
\hline Clinical tumor size & 1.15 & 0.15 & $0.95-1.4$ & 1.5 & 0.22 & $0.9-1.5$ \\
\hline Charlson index $\geq 2$ & 0.5 & 0.1 & $0.2-1.15$ & 0.6 & 0.46 & $0.19-2.13$ \\
\hline Imperative indication & 3.9 & 0.050 & $1.0-16.0$ & 3.1 & 0.21 & $0.5-20.1$ \\
\hline PADUA score $\geq 10$ & 0.9 & 0.90 & $0.25-3.4$ & 1.04 & 0.96 & $0.17-6.1$ \\
\hline Technique open versus robotic & 3.8 & 0.005 & $1.5-9.5$ & 9.4 & 0.03 & $1.2-72.9$ \\
\hline Use of ischemia & 0.9 & 0.78 & $0.5-1.8$ & 0.9 & 0.46 & $0.19-2.13$ \\
\hline
\end{tabular}

$P A D U A$ preoperative aspects and dimensions used for anatomical classification of renal tumor

However, a previous study on this issue by Lee and colleagues reported a longer WIT in the RPN group (23 vs. $19 \min , p<0.001)$ [11]. It is not easy to justify this heterogeneity of results. Surely, the increasing surgeons experience in RPN may lead to a progressive reduction in WIT in the most recent series.

Interestingly, in the present series that evaluated all cases of PN regardless the nephrometry score, the robotic approach also resulted in a significant reduction of postoperative surgical morbidity. This is not in agreement with Simhan, which in tumors with moderate-high complexity according to RENAL score reported comparable postoperative complications [9]. This might suggest that benefits in terms of surgical morbidity of RPN are best expressed in tumors of low complexity, although this hypothesis cannot be supported by ourselves. In fact, in a subanalysis of patients with moderate-high nephrometry (PADUA score $\geq 8$ ), not shown in the results section, the advantages of RPN in terms of overall postoperative complications remained significant ( 8 vs. $29.9 \%$; $p=0.026$ ). A possible explanation for the reduction of complications might be the better visualization of the resection bed in resulting of a better closure of vessels and/or urinary collecting system. Prospective studies with larger series are needed to further elucidate this issue, and to confirm whether or not RPN has a lower surgical morbidity than OPN, as previously shown in comparison with LPN [6]. In our study, the advantages of RPN regarding the surgical morbidity could have been partially overestimated, due to a not negligible postoperative complications rate in the OPN group (24.2\%) and to a favorable complication rate in the RPN group that are, however, within the ranges reported in the literature (9.6-30.7 and 8-22\% for OPN and RPN, respectively) [2226]. Also our rate of acute or delayed postoperative hemorrhage requiring reintervention (3\%) and of urinary fistulas $(5.5 \%)$ after OPN are within the ranges reported in the literature, $1.3-7.9 \%[24,27,28]$ and $1.4-17.4 \%[23,29]$, respectively. Also Lee et al., in their retrospective study of OPN versus RPN, reported a higher overall postoperative complications rate in the open group (15.4 and $8.7 \%$, respectively; $p=0.158$ ) although without reaching the statistical significance, possibly due to the relatively low number of patients in RPN group $(n=69)$. None of the previous comparisons between OPN and RPN subdivided postoperative complications into medical and surgical; in our series, the difference in postoperative complications was solely related to surgical morbidity, while no difference in medical complications was found, although RPN group had an higher Charlson index at baseline.

The oncological outcomes of RPN are usually presented using surrogate endpoints such as the PSM rate. Our PSM rate in RPN group was not higher than OPN, and both were within the range reported in the literature $(0-7 \%)$.

We acknowledge some limitations to the present study. First, surgeons experience was higher in the open group. This is an intrinsic bias of studies, which compare a new technique with the standard treatment. Moreover, tumor diameter was higher in OPN group. Nevertheless, tumor nephrometry was homogeneous, and in previous analyses, it resulted a stronger predictor of complications compared to tumor diameter [30]. The strengths of the present study are inherent to the prospective data collection, to the multicenter nature, to the strict definition of any complication that was decided before the data recording, and to the separate analysis of medical and surgical complications. This approach might increase the external validity of the data compared with the single-center, single-surgeon setting, reflecting a "real world" scenario.

The present experience evaluated the perioperative results only; future studies with appropriate follow-up will assess the differences of oncological and long-term functional outcomes between RPN and OPN.

\section{Conclusion}

In our analysis, RPN resulted comparable to OPN in terms of WIT and PSM rate, and showed a reduction of bleeding, 
postoperative surgical complications, and hospitalization. Operative time was significantly higher in RPN group.

Conflict of interest None.

\section{References}

1. Ljungberg B, Cowan NC, Hanbury DC et al. (2010) European association of urology guideline group. EAU guidelines on renal cell carcinoma: the 2010 update. Eur Urol 58(3):398-406. Epub 2010 Jul 12. PubMed PMID: 20633979

2. Novick AC, Campbell SC, Belldegrun A et al: Guideline for management of the clinical stage 1 renal mass. Available at www. auanet.org/content/ media/renalmass09.pdf?CFID 1666475\& CFTOKEN 79651347\&jsessionid 8430e85e87e2ad1bac0325294 a7325226072. Accessed 1 Aug 2010

3. Antonelli A, Ficarra V, Bertini R et al (2012) Members of the SATURN Project-LUNA Foundation. Elective partial nephrectomy is equivalent to radical nephrectomy in patients with clinical T1 renal cell carcinoma: results of a retrospective, comparative, multi-institutional study. BJU Int 109(7):1013-1018

4. Huang WC, Elkin EB, Levey AS et al (2009) Partial nephrectomy versus radical nephrectomy in patients with small renal tumors-is there a difference in mortality and cardiovascular outcomes? J Urol 181(1):55-61

5. Miller DC, Schonlau M, Litwin MS et al (2008) Urologic diseases in America project. Renal and cardiovascular morbidity after partial or radical nephrectomy. Cancer 112(3):511-520

6. Khalifeh A, Autorino R, Hillyer SP et al. (2013) Comparative outcomes and assessment of "Trifecta" in 500 robotic and laparoscopic partial nephrectomies: a single surgeon experience. J Urol 189(4):1236-1242

7. Benway BM, Bhayani SB, Rogers CG et al (2009) Robot assisted partial nephrectomy versus laparoscopic partial nephrectomy for renal tumors: a multi-institutional analysis of perioperative outcomes. J Urol 182(3):866-872

8. Pierorazio PM, Patel HD, Feng T, et al. (2011) Robotic-assisted versus traditional laparoscopic partial nephrectomy: comparison of outcomes and evaluation of learning curve. Urology 78(4):813-819. Epub 2011 Jul 29

9. Simhan J, Smaldone MC, Tsai KJ et al (2012) Perioperative outcomes of robotic and open partial nephrectomy for moderately and highly complex renal lesions. J Urol 187(6):2000-2004

10. Sprenkle PC, Power N, Ghoneim T et al (2012) Comparison of open and minimally invasive partial nephrectomy for renal tumors 4-7 centimeters. Eur Urol 61(3):593-599

11. Lee S, Oh J, Hong SK, et al. (2011) Open versus robot-assisted partial nephrectomy: effect on clinical outcome. J Endourol 25(7):1181-1185. Epub 2011 Jun 9

12. Mottrie A, De Naeyer G, Schatteman P et al (2010) Impact of the learning curve on perioperative outcomes in patients who underwent robotic partial nephrectomy for parenchymal renal tumours. Eur Urol 58(1):127-132

13. Ficarra V, Novara G, Secco S et al (2009) Preoperative aspects and dimensions used for an anatomical (PADUA) classification of renal tumours in patients who are candidates for nephronsparing surgery. Eur Urol 56(5):786-793
14. Levey AS, Stevens LA, Schmid CH et al (2009) A new equation to estimate glomerular filtration rate. Ann Intern Med 150(9): 604-612

15. Greene FL, Gospodarowicz M, Wittekend C et al (2009) American joint committee on cancer (AJCC) staging manual, 7th edn. Springer, Philadelphia

16. Fuhrman SA, Lasky LC, Limas C (1982) Prognostic significance of morphologic parameters in renal cell carcinoma. Am J Surg Pathol 6:655-663

17. Eble JN, Sauter G, Epstein JI et al (2004) Pathology and genetics of tumours of the urinary system and male genital organs. World health organization classification of tumours. IARC Press, Lyon

18. Dindo D, Demartines N, Clavien PA (2004) Classification of surgical complications: a new proposal with evaluation in a cohort of 6336 patients and results of a survey. Ann Surg 240:205-213

19. Wang AJ, Bhayani SB (2009) Robotic partial nephrectomy versus laparoscopic partial nephrectomy for renal cell carcinoma: singlesurgeon analysis of 100 consecutive procedures. Urology 73: 306-310

20. Kural AR, Atug F, Tufek I et al (2009) Robot-assisted partial nephrectomy versus laparoscopic partial nephrectomy: comparison of outcomes. J Endourol 23:1491-1497

21. Rogers CG, Patard JJ (2009) Rebuttal 2 open to debate. The motion: robotic partial nephrectomy is better than open partial nephrectomy. Eur Urol 56(3):568-570. Epub 2009 Jun 24

22. Pasticier G, Timsit MO, Badet L et al (2006) Nephron-sparing surgery for renal cell carcinoma: detailed analysis of complications over a 15-year period. Eur Urol 49(3):485-490

23. Campbell SC, Novick AC, Streem SB et al (1994) Complications of nephron sparing surgery for renal tumors. J Urol 151(5): $1177-1180$

24. Thompson RH, Leibovich BC, Lohse CM et al (2005) Complications of contemporary open nephron sparing surgery: a single institution experience. J Urol 174(3):855-858

25. Dulabon LM, Kaouk JH, Haber GP et al (2011) Multi-institutional analysis of robotic partial nephrectomy for hilar versus nonhilar lesions in 446 consecutive cases. Eur Urol 59(3): 325-330

26. Gupta GN, Boris R, Chung P et al (2011) Robot-assisted laparoscopic partial nephrectomy for tumors greater than $4 \mathrm{~cm}$ and high nephrometry score: feasibility, renal functional, and oncological outcomes with minimum 1 year follow-up. Urol Oncol 31:51-56

27. Van Poppel H, Bamelis B, Oyen R, Baert L (1998) Partial nephrectomy for renal cell carcinoma can achieve long-term tumor control. J Urol 160(3 Pt 1):674-678

28. Stephenson AJ, Hakimi AA, Snyder ME, Russo P (2004) Complications of radical and partial nephrectomy in a large contemporary cohort. J Urol 171(1):130-134

29. Pomara G, Campo G, Francesca F (2009) Intraoperative and postoperative complications of nephron sparing surgery: prevention and possible treatments. Arch Ital Urol Androl 81(2):80-85 Review

30. Minervini A, Vittori G, Salvi M et al (2013) Analysis of surgical complications of renal tumor enucleation with standardized instruments and external validation of PADUA classification. Ann Surg Oncol 20(5):1729-1736 